\title{
A NOTE ON ENVELOPED MEASURES
}

\section{S. J. SIDNEY}

\begin{abstract}
There exists a uniform algebra $\mathfrak{U}$ and a multiplicative linear functional $\Phi$ on $\mathfrak{I}$ which has an uncountable family of enveloped representing measures, no one of which is boundedly absolutely continuous with respect to any other.
\end{abstract}

Gamelin and Lumer have asked whether two enveloped representing measures for the same multiplicative linear functional on a uniform algebra must be mutually boundedly absolutely continuous [2, p. 142]. In the present note, a uniform algebra $\mathfrak{U}$ and multiplicative linear functional $\Phi$ constructed previously by the author [4] are shown to have the following property: $\Phi$ has an uncountable family of enveloped representing measures, no one of which is boundedly absolutely continuous with respect to any other. We retain the notations and conventions of [4].

Suppose $\left\{C_{n}\right\}_{1 \leqq n<\infty}$ is any sequence of constants which satisfies

$$
C_{n}>0 \text { and } \sum_{n=1}^{\infty} C_{n} P_{n} \leqq \beta
$$

for some number $\beta<1$. We assert that the corresponding $\mu \in M_{\Phi}$ is enveloped. Indeed, let $\left\{u^{(k)}\right\}$ be a sequence of nonnegative continuous functions on $X$ such that $\int u^{(k)} d \mu \rightarrow 0$. It suffices to show that, given $0<\delta<1$, we can find for all $k$ large enough $f^{(k)} \in \mathfrak{A}$ such that $\left|f^{(k)}\right| \leqq$ $\exp \left(-u^{(k)}\right)$ but $\left|\Phi\left(f^{(k)}\right)\right|>(1-\delta)^{2} e^{-\delta}$.

Since $\left(1-\left|Z_{n}\right|\right)\left(1+\left|Z_{n}\right|\right)^{-1} \leqq P_{n}$ we see from (4) of [4] that $\sum\left(1-\left|Z_{n}\right|\right)<$ $\infty$; hence if we fix an integer $N$ large enough, the Blaschke product

$$
B(z)=\prod_{n=N^{+}+1}^{\infty}\left(Z_{n}\left|Z_{n}\right|^{-1}\right)\left(Z_{n}-z\right)\left(1-Z_{n} z\right)^{-1}
$$

will be continuous and bounded by 1 on $\Delta \backslash\{1\}$, holomorphic on the interior of $\Delta$, and such that $B(0)>1-\delta$.

Let $S=\bigcup_{n=0}^{N} T_{n}, \mathfrak{B}=\mathfrak{X} \mid S, v$ the measure on $S$ given by $\nu_{n}=\mu_{n}=$ $C_{n} P_{n} \lambda, 1 \leqq n \leqq N$, and $v_{0}=\left(1-\sum_{n=1}^{N} C_{n} P_{n}\right) \lambda$. One sees easily that $\mathfrak{B}$ is a uniform algebra on $S$ and that $v$ is a core measure for $\Phi$ (given again by

Received by the editors March 1, 1972.

AMS (MOS) subject classifications (1969). Primary 4655.

Key words and phrases. Enveloped measure, uniform algebra, multiplicative linear functional, Blaschke product, core measure.

(c) American Mathematical Society 1973 
$\left.\Phi(f)=\hat{f}_{0}(0)\right)$. Viewed as a measure on $X, v \leqq(1-\beta)^{-1} \mu$, so $\int u^{(k)} d v \rightarrow 0$. Thus by [1, IV.5.1] for $k$ large there is $g^{(k)}=v^{(k)}+i w^{(k)} \in \mathfrak{B}$ such that $v^{(k)} \geqq u^{(k)} \mid S$ and $\Phi\left(g^{(k)}\right)<\delta$. Finally, there is $h$ in the disc algebra $A$ such that $|h| \leqq 1, h(1)=0$, and $|\hat{h}(0)-1|<\delta$. Define $f^{(k)}: X \rightarrow C$ by $f_{n}^{(k)}=$ $h B \exp \left(-g_{n}^{(k)}\right), \quad 0 \leqq n \leqq N$, and $f_{n}^{(k)}=0, N+1 \leqq n \leqq \infty$. Then $f^{(k)} \in \mathfrak{A}$, $\left|f^{(k)}\right| \leqq \exp \left(-u^{(k)}\right)$, and $\left|\Phi\left(f^{(k)}\right)\right|=|\hat{h}(0)||B(0)| \exp \left(-\Phi\left(g_{n}^{(k)}\right)\right)>(1-\delta)^{2} e^{-\delta}$.

Suppose now that $\left\{\bar{C}_{n}\right\}$ is any sequence that satisfies $\left(^{*}\right)$ (for instance, the sequence $\left\{\widetilde{C}_{n}\right\}$ of [4]). If $\left\{\tau_{n}\right\}_{1 \leqq n}$ is any sequence of numbers such that $0<\tau_{n} \leqq 1$, then $\left\{\tau_{n} \bar{C}_{n}\right\}$ also satisfies $\left({ }^{*}\right)$. Now, there is an uncountable family $\Omega$ of infinite sets of positive integers such that any two members of $\Omega$ have finite intersection [3, Problem 5I]. For $\omega \in \Omega$ let $\tau_{n}^{\omega}=1 / n$ if $n \in \omega$, $\tau_{n}^{\omega}=1$ if $n \notin \omega$. If $\omega, \omega^{\prime}$ are distinct members of $\Omega$, one sees easily that neither of the measures corresponding to the sequences $\left\{\tau_{n}^{\omega} \bar{C}_{n}\right\}$ and $\left\{\tau_{n}^{\omega^{\prime}} \bar{C}_{n}\right\}$ is boundedly absolutely continuous with respect to the other.

REMARKS. Evidently the present arguments do not require the special choice of $\left\{Z_{n}\right\}$ necessary in [4], but rather only that $Z_{n} \rightarrow 1$ and $\sum\left(1-\left|Z_{n}\right|\right)<\infty$. Under these conditions, it is easy to see that $M_{\Phi}$ is necessarily compact in norm. (This also follows immediately from a more difficult unpublished result of $\mathrm{J}$. Chaumat about norm-compactness of spaces of representing measures for the disc algebra.) In particular, by taking the $Z_{n}$ positive and increasing rapidly to 1 , we can arrange that $M_{\Phi}$ be norm-compact and contain many enveloped measures, but not contain a core measure. The converse question, of whether norm-compactness of $M_{\Phi}$ is a consequence of the existence of a core measure, appears to be open. I wish to thank A. Dufresnoy for pointing out to me that $M_{\Phi}$ is normcompact.

\section{REFERENCES}

1. T. W. Gamelin, Uniform algebras, Prentice-Hall, Englewood Cliffs, N.J., 1969.

2. T. W. Gamelin and G. Lumer, Theory of abstract Hardy spaces and the universal Hardy class, Advances in Math. 2 (1968), 118-174. MR 37 \#1982.

3. L. Gillman and M. Jerison, Rings of continuous functions, University Series in Higher Math., Van Nostrand, Princeton, N.J., 1960. MR 22 \#6994.

4. S. J. Sidney, An example concerning core measures, Proc. Amer. Math. Soc. 26 (1970), 428-430. MR 41 \#8997.

Department of Mathematics, University of Connecticut, Storrs, Connecticut 06268

Université Scientifique et Médicale de Grenoble, Grenoble, France 